Supporting Information

\title{
Volatile Acid Responsiveness of Chiral Nematic Luminescent Cellulose Nanocrystal/9,10-Bis((Z)-2-phenyl-2-(pyridin-2-yl) vinyl)anthracene Composite Films
}

Jintao He, Kaiqiang Bian, $\mathrm{Na} \mathrm{Li,} \mathrm{and} \mathrm{Guangzhe} \mathrm{Piao*}$

Key Laboratory of Rubber-Plastics, Ministry of Education/Shandong Provincial

Key Laboratory of Rubber-Plastics, Qingdao University of Science \&

Technology, Qingdao 266042, China

Tel.: +86-0532-84022725; Fax: +86-532-840-22725

E-mail address: piao@qust.edu.cn (G. Piao) 


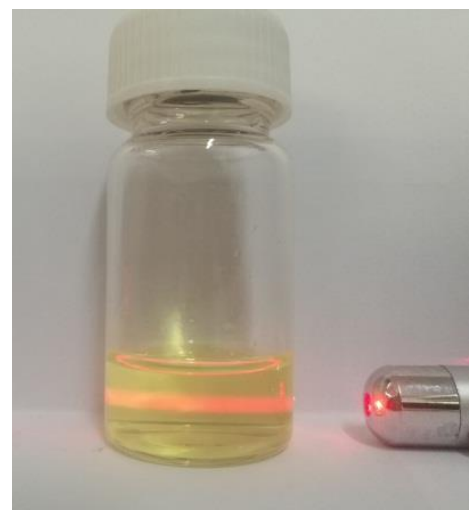

Figure. S1 The Tyndall phenomenon of BPP2VA suspension.

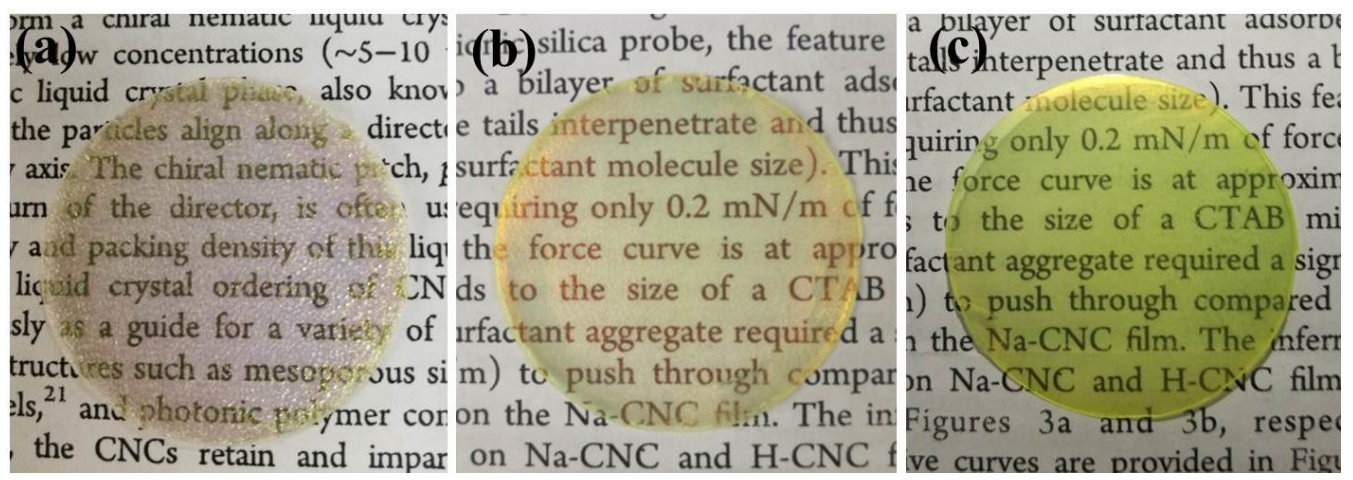

Figure. S2 Photographs of CNC-BPP2VA composite films: (a) CNC-BPP2VA-1, (b)

CNC-BPP2VA-2 and (c) CNC-BPP2VA-3.
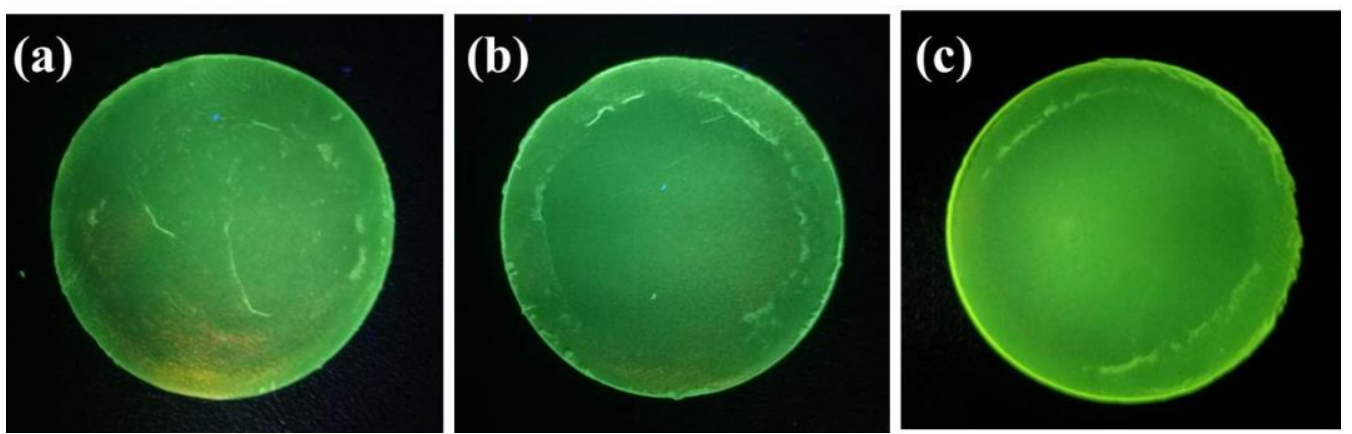

Figure. S3 Photographs of CNC-BPP2VA composite films under the UV-365nm light

irradiation: (a) CNC-BPP2VA-1, (b) CNC-BPP2VA-2 and (c) CNC-BPP2VA-3. 


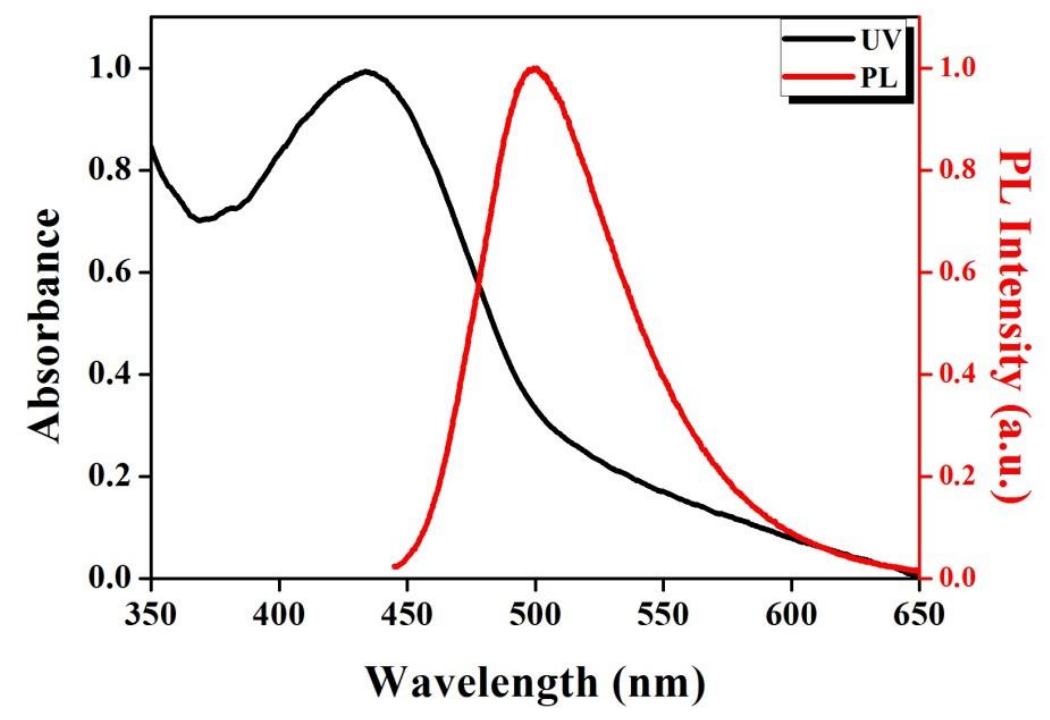

Figure. S4 The spectra of UV-vis and fluorescence measured from the BPP2VA in THF solution, $\lambda_{\mathrm{ex}}=425 \mathrm{~nm}$.
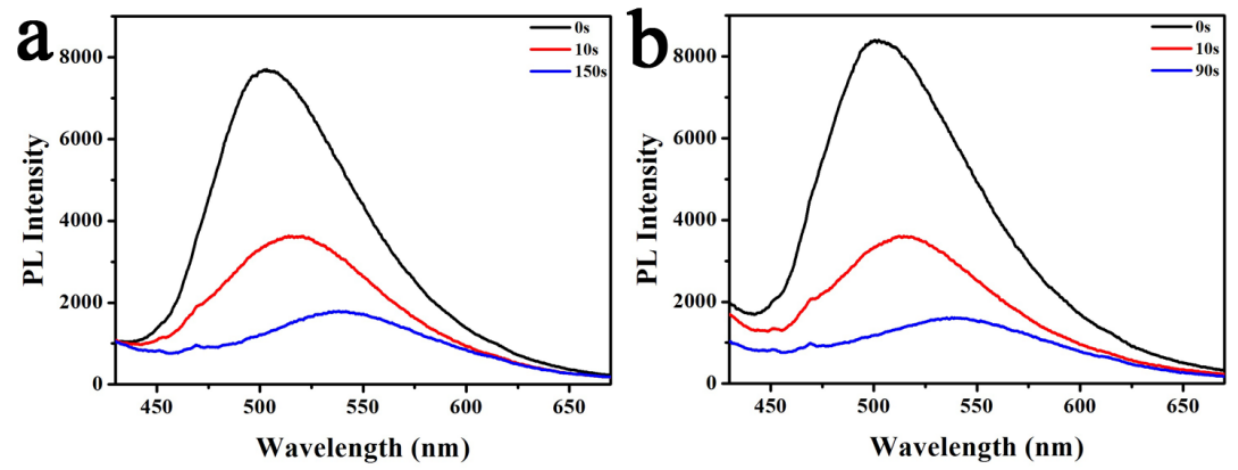

Figure. S5 Fluorescence spectra of CNC-BPP2VA-3composite film upon exposure to

$\mathrm{HCl}$ vapors with a concentration of (a) $10 \mathrm{ppm}$ and (b)100 ppm, $\lambda_{\mathrm{ex}}=405 \mathrm{~nm}$.
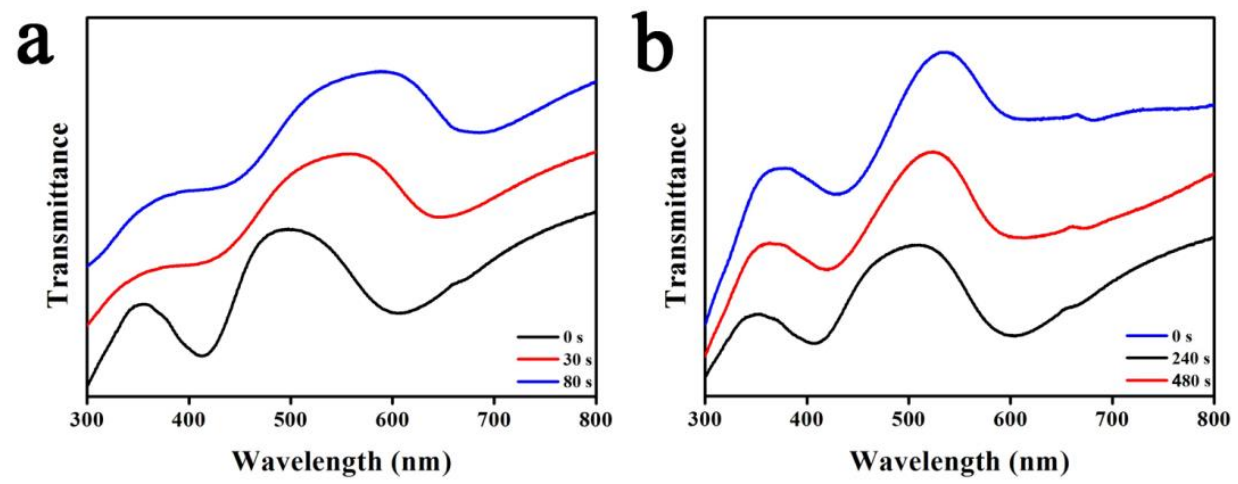

Figure. S6 UV-vis spectra of CNC-BPP2VA-1 composite film upon exposure to (a) TFA and (b) $\mathrm{HNO}_{3}$ vapor with different times. 


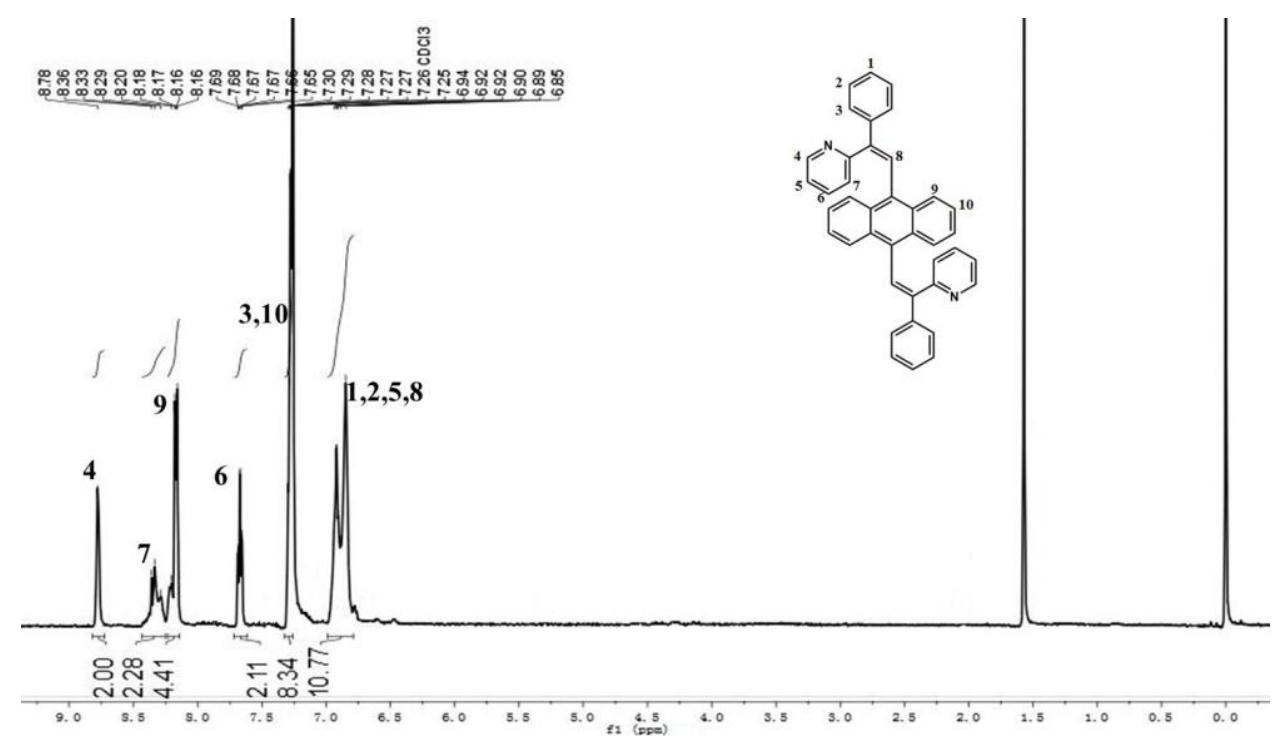

Figure. $\mathbf{S} 7{ }^{1} \mathrm{H}$ NMR spectrum of BPP2VA $\left(500 \mathrm{MHz}, \mathrm{CDCl}_{3}\right.$, TMS, $\left.25{ }^{\circ} \mathrm{C}\right)$. 